Theor Appl Genet (1986) 72:433-439

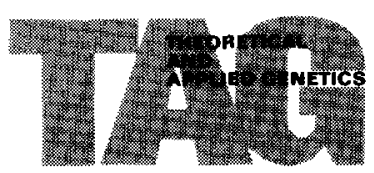

(C) Springer-Verlag 1986

\title{
Utilization of wild relatives in the genetic improvement of Arachis hypogaea L.
}

\section{Synthetic amphidiploids and their importance in interspecific breeding *}

\author{
A. K. Singh \\ Groundnut Improvement Program, ICRISAT, Patancheru P.O.-502 324, A.P., India \\ Received January 20, 1986; Accepted February 27, 1986 \\ Communicated by J.MacKey
}

Summary. Synthetic amphidiploids were established in 32 combinations involving 8 diploid wild species representing both A and B genomes of section Arachis. Bivalent and multivalent associations in the amphidiploids of $7 \mathrm{~A}$ genome species confirm that these species have identical genomes. Contrastingly, high bivalent frequencies in amphidiploids involving the $\mathrm{A}$ and $\mathrm{B}$ genome species suggest that $A$. batizoco $i$ has a distinct ' $\mathrm{B}$ ' genome that is partially homologous to the other genome ' $A$ ' represented in the rest of the species. Crossability, chromosome pairing and pollen and pod fertility in hybrids between $A$. hypogaea and amphidiploids have revealed that these amphidiploids can be used as a genetic bridge for the transfer of genes from the wild species into the cultivated groundnut.

Key words: Arachis - Amphidiploids - Genomes Chromosome pairing - Recombinations

\section{Introduction}

The section Arachis of genus Arachis includes the tetraploid cultivated species $A$. hypogaea and several wild diploid species. These wild species have resistance to several important pests and pathogens of groundnut (Abdou et al. 1974; Subrahmanyam et al. 1985; Amin 1985) and can be used for the genetic improvement of groundnut. The $A$. hypogaea is a segmental allotetraploid made up of two $\mathrm{A}$ and two $\mathrm{B}$ genomes (Husted 1936; Singh and Moss 1984). The A genome is found in several diploid species while the B genome is present

* Submitted as Journal Article No. 530 by International Crops Research Institute for the Semi-Arid Tropics (ICRISAT) only in A. batizocoi (Smartt et al. 1978). Based on karyomorphological differences, the diploid species have been divided into two clusters (Singh and Moss 1982). This genomic relationship between wild and cultivated species suggests that hybridization between synthetic amphidiploids of wild species and $A$. hypogaea should be a promising approach for the transfer of desirable genes from wild species into $A$. hypogaea (Stalker and Wynne 1979; Moss 1980; Gardner and Stalker 1983; Singh and Moss 1984; Singh 1985).

This paper reports on the production of synthetic amphidiploids involving several $\mathrm{A}$ genome species and A. batizocoi, and on the cytological behaviour of hybrids produced from crosses between $A$. hypogaea and these amphidiploids. The performance of these amphidiploids as a genetic bridge for introgression from the wild species into the cultivated groundnut is discussed.

\section{Materials and methods}

The identities and sources of the eight diploid wild species and the cultivars of $A$. hypogaea used in this investigation have already been described by Singh and Moss (1984).

The $F_{1}$ seedlings of the 34 diploid hybrid combinations of a possible 56 combinations from 8 diploid wild species and two complex hybrids: (i) $A$. species HLK $410 \times(A$. chacoense $\times A$. cardenasii) and (ii) $A$. correntina $\times(A$. chacoense $\times A$. cardenasii) were treated with $0.25 \%$ and $0.35 \%$ colchicine solution (Singh 1986). Amphidiploids were established in 32 of the 36 combinations. Of these, 22 amphidiploids, including both intracluster (A, A) and intercluster (A, B) species, were crossed as males with one or more cultivars belonging to either of the two subspecies of $A$. hypogaea. The $A$. batizocoi $\times A$. chacoense amphidiploid was also crossed as female. $F_{1}$ hybrids were established with all 22 amphidiploids.

Hybridization, and cytological and pollen fertility analyses were done according to Singh and Moss (1984). Chromosome associations were statistically analysed using the one-way analysis of variance (Cochran and Cox 1957). 
Table 1. Chromosome associations at diakinesis/metaphase I in synthetic amphidiploids from diploid species of section Arachis genus Arachis

\begin{tabular}{|c|c|c|c|c|c|c|c|}
\hline \multirow[t]{2}{*}{ Amphidiploids } & \multirow{2}{*}{$\begin{array}{l}\text { No. of } \\
\text { cells } \\
\text { analysed }\end{array}$} & \multicolumn{4}{|c|}{ Chromosome association } & \multirow{2}{*}{$\begin{array}{l}\text { No. of } \\
\text { chiasmata }\end{array}$} & \multirow{2}{*}{$\begin{array}{l}\text { No. of ter- } \\
\text { minalised } \\
\text { chiasmata }\end{array}$} \\
\hline & & I & II & III & IV & & \\
\hline \multicolumn{8}{|l|}{ Synthetic amphidiploid, $C_{1}$} \\
\hline \multicolumn{8}{|l|}{ Intercluster (AB) } \\
\hline $\begin{array}{l}\text { batizocoi } \times \text { duranensis } \\
\text { batizocoi } \times \text { chacoense } \\
\text { batizocoi } \times \text { correntina } \\
\text { correntina } \times \text { batizocoi } \\
\text { batizoco } \times \text { villosa }\end{array}$ & $\begin{array}{l}19 \\
22 \\
10 \\
11 \\
25\end{array}$ & $\begin{array}{l}2.5 \pm 0.33 \\
1.5 \pm 0.44 \\
4.5 \pm 0.75 \\
2.0 \pm 0.54 \\
6.1 \pm 0.68\end{array}$ & $\begin{array}{l}16.6 \pm 0.45 \\
15.6 \pm 0.42 \\
16.6 \pm 0.64 \\
17.6 \pm 0.55 \\
15.3 \pm 0.50\end{array}$ & $\begin{array}{l}0.5 \pm 0.19 \\
0.1 \pm 0.06 \\
0.5 \pm 0.27 \\
0.2 \pm 0.15 \\
0.3 \pm 0.11\end{array}$ & $\begin{array}{l}0.7 \pm 0.17 \\
1.8 \pm 0.20 \\
0.2 \pm 0.13 \\
0.3 \pm 0.16 \\
0.6 \pm 0.14\end{array}$ & $\begin{array}{l}33.9 \pm 0.64 \\
36.4 \pm 0.53 \\
- \\
30.0 \pm 0.87 \\
-\end{array}$ & $\begin{array}{l}32.6 \pm 0.60 \\
35.4 \pm 0.52 \\
- \\
28.0 \pm 0.60 \\
-\end{array}$ \\
\hline \multicolumn{8}{|l|}{ Intracluster $(\mathrm{AA})$} \\
\hline $\begin{array}{l}\text { sp. GKP } 10038 \times \text { duranensis } \\
\text { sp. GKP } 10038 \times \text { sp. HLK } 410 \\
\text { duranensis } \times \text { sp. GKP } 10038 \\
\text { duranensis } \times \text { cardenasii } \\
\text { duranensis } \times \text { correntina } \\
\text { duranensis } \times \text { villosa } \\
\text { sp. HLK } 410 \times \text { sp. GKP } 10038 \\
\text { sp. HLK } 410 \times \text { chacoense } \\
\text { sp. HLK } 410 \times \text { correntina } \\
\text { sp. HLK } 410 \times \text { villosa } \\
\text { sp. HLK } 410 \times \\
(\text { chacoense } \times \text { cardenasii })\end{array}$ & $\begin{array}{l}25 \\
16 \\
13 \\
30 \\
21 \\
20 \\
31 \\
15 \\
25 \\
23 \\
23\end{array}$ & $\begin{array}{l}0.9 \pm 0.28 \\
1.9 \pm 0.38 \\
0.9 \pm 0.42 \\
1.7 \pm 0.31 \\
2.1 \pm 0.42 \\
2.0 \pm 0.52 \\
3.5 \pm 0.53 \\
0.5 \pm 0.26 \\
3.0 \pm 0.62 \\
1.0 \pm 0.25 \\
3.0 \pm 0.81\end{array}$ & $\begin{array}{l}13.0 \pm 0.50 \\
15.1 \pm 0.97 \\
11.7 \pm 0.96 \\
13.3 \pm 0.58 \\
11.8 \pm 0.75 \\
13.6 \pm 0.56 \\
14.0 \pm 0.45 \\
14.6 \pm 0.72 \\
15.2 \pm 0.41 \\
14.3 \pm 0.61 \\
13.3 \pm 0.64\end{array}$ & $\begin{array}{l}0.2 \pm 0.13 \\
0.4 \pm 0.15 \\
0.1 \pm 0.08 \\
0.3 \pm 0.08 \\
0.3 \pm 0.17 \\
0.1 \pm 0.05 \\
0.4 \pm 0.11 \\
0.6 \pm 0.24 \\
0.3 \pm 0.13 \\
0.4 \pm 0.12 \\
0.7 \pm 0.16\end{array}$ & $\begin{array}{l}3.0 \pm 0.23 \\
1.6 \pm 0.43 \\
3.7 \pm 0.47 \\
2.6 \pm 0.23 \\
3.3 \pm 0.38 \\
2.6 \pm 0.29 \\
1.8 \pm 0.19 \\
2.1 \pm 0.34 \\
1.4 \pm 0.20 \\
2.2 \pm 0.29 \\
2.1 \pm 0.25\end{array}$ & $\begin{array}{c}35.6 \pm 0.50 \\
35.8 \pm 0.64 \\
- \\
35.9 \pm 0.57 \\
36.5 \pm 0.46 \\
33.2 \pm 0.65 \\
34.0 \pm 0.63 \\
37.0 \pm 0.47 \\
32.0 \pm 0.65 \\
37.3 \pm 0.42 \\
32.8 \pm 1.46\end{array}$ & $\begin{array}{c}35.5 \pm 0.50 \\
34.1 \pm 0.58 \\
- \\
33.9 \pm 0.65 \\
36.2 \pm 0.51 \\
33.0 \pm 0.69 \\
34.7 \pm 0.60 \\
35.4 \pm 0.57 \\
31.8 \pm 0.65 \\
37.2 \pm 0.45 \\
32.1 \pm 1.41\end{array}$ \\
\hline $\begin{array}{l}\text { correntina } \times \text { sp. HLK } 410 \\
\text { correntina } \times \text { chacoense } \\
\text { correntina } \times \text { villosa } \\
\text { correntina } \times \\
(\text { chacoense } \times \text { cardenasii })\end{array}$ & $\begin{array}{l}13 \\
11 \\
24 \\
10\end{array}$ & $\begin{array}{l}4.5 \pm 0.94 \\
3.6 \pm 0.73 \\
3.3 \pm 0.46 \\
2.7 \pm 1.07\end{array}$ & $\begin{array}{l}15.2 \pm 0.58 \\
14.3 \pm 0.56 \\
13.5 \pm 0.73 \\
14.9 \pm 0.94\end{array}$ & $\begin{array}{l}0.1 \pm 0.08 \\
0.6 \pm 0.20 \\
0.4 \pm 0.10 \\
0.5 \pm 0.31\end{array}$ & $\begin{array}{l}1.2 \pm 0.34 \\
1.60 \pm 0.28 \\
2.1 \pm 0.32 \\
1.5 \pm 0.37\end{array}$ & $\begin{array}{l}33.2 \pm 0.96 \\
32.7 \pm 1.03 \\
31.1 \pm 0.78 \\
-\end{array}$ & $\begin{array}{l}32.9 \pm 0.88 \\
32.6 \pm 1.11 \\
31.1 \pm 0.73 \\
-\end{array}$ \\
\hline $\begin{array}{l}\text { villosa } \times \text { duranensis } \\
\text { villosa } \times \text { sp. HLK } 410\end{array}$ & $\begin{array}{r}6 \\
25\end{array}$ & $\begin{array}{l}1.0 \pm 0.45 \\
2.6 \pm 0.50\end{array}$ & $\begin{array}{l}14.33 \pm 1.26 \\
13.6 \pm 0.62\end{array}$ & $\begin{array}{l}0.3 \pm 0.33 \\
0.7 \pm 0.14\end{array}$ & $\begin{array}{l}2.3 \pm 0.71 \\
2.0 \pm 0.31\end{array}$ & $\begin{array}{l}35.1 \pm 0.56 \\
32.3 \pm 1.20\end{array}$ & $\begin{array}{l}35.2 \pm 0.31 \\
32.0 \pm 1.53\end{array}$ \\
\hline \multicolumn{8}{|l|}{ Synthetic amphidiploid, $\mathrm{C}_{2}$} \\
\hline $\begin{array}{l}\text { correntina } \times \text { batizocoi } \\
\text { villosa } \times \text { batizocoi } \\
\text { sp. GKP } 10038 \times \text { duranensis } \\
\text { sp. GKP } 10038 \times \text { sp. HLK } 410 \\
\text { correntina } \times \text { sp. HLK } 410 \\
\text { villosa } \times \text { duranensis }\end{array}$ & $\begin{array}{r}4 \\
25 \\
20 \\
14 \\
9 \\
9\end{array}$ & $\begin{array}{l}0.5 \pm 0.29 \\
1.5 \pm 0.31 \\
1.45 \pm 0.30 \\
1.0 \pm 0.39 \\
3.9 \pm 0.84 \\
0.4 \pm 0.44\end{array}$ & $\begin{array}{l}16.0 \pm 0.41 \\
18.2 \pm 0.33 \\
16.45 \pm 0.43 \\
14.6 \pm 0.88 \\
14.0 \pm 0.85 \\
15.8 \pm 0.78\end{array}$ & $\begin{array}{l}1.5 \pm 0.65 \\
0.4 \pm 0.14 \\
0.35 \pm 0.13 \\
0.2 \pm 0.11 \\
0.8 \pm 0.15 \\
0.0 \pm 0.00\end{array}$ & $\begin{array}{l}0.8 \pm 0.48 \\
0.2 \pm 0.08 \\
1.15 \pm 0.17 \\
2.2 \pm 0.39 \\
1.4 \pm 0.41 \\
2.0 \pm 0.33\end{array}$ & $\begin{array}{l}36.0 \pm 1.41 \\
35.4 \pm 0.29 \\
38.0 \pm 0.42 \\
37.1 \pm 0.67 \\
34.7 \pm 0.93 \\
39.1 \pm 0.54\end{array}$ & $\begin{array}{l}34.0 \pm 2.68 \\
34.8 \pm 0.31 \\
33.65 \pm 0.63 \\
35.7 \pm 0.73 \\
34.7 \pm 0.93 \\
38.2 \pm 0.57\end{array}$ \\
\hline
\end{tabular}

\section{Results and discussion}

\section{Synthetic amphidiploids, $C_{1}$}

The established amphidiploids were more vigorous than their parent species or the straight hybrids. Cytological analysis of 5 intercluster AABB amphidiploids and 17 intracluster AAAA amphidiploids showed relatively higher bivalent frequencies for intercluster amphidiploids than for the intracluster amphidiploids (Table 1; Figs. 1 and 2). The multivalent frequencies observed for the AAAA amphidiploids (Table 1) were comparable to those of the genome A autotetraploids (Singh 1986), confirming the identical genomic constitution of A genome species of the section Arachis. However, Gardner and Stalker (1983) recorded a comparatively higher bivalent association in 14 of these AAAA amphidiploids involving the same 6 genome A species of section Arachis. These conflicting results are difficult to explain based on normal association observed in the diploid $F_{1}$ hybrids of these species (Stalker and Wynne 1979; Singh and Moss 1984) unless there is some pairing control mechanism functional at the tetraploid level with environmental dependence (temperature, etc.).

The significantly higher bivalent association (Table 1) and consequently more normal PMCs in intercluster amphidiploids suggests their segmental allopolyploid nature. However, considerable variation in pollen and pod fertility was observed among plants of both inter- and intracluster amphidiploids (Table 2). Such differences between sister plants can be attributed to disturbances in the karyotypic, genetic and/or physiological balance. 
Table 2. Percentage of different chromosomal distributions at Anaphase I, and pollen and pod fertility in synthetic amphidiploids of diploid species of section Arachis genus Arachis

\begin{tabular}{|c|c|c|c|c|c|c|c|}
\hline \multirow[t]{2}{*}{ Amphidiploid } & \multirow{2}{*}{$\begin{array}{l}\text { No. of } \\
\text { cells } \\
\text { analysed }\end{array}$} & \multicolumn{4}{|c|}{ Chromosomal distribution at $\mathrm{AI}$} & \multirow{2}{*}{$\begin{array}{l}\% \text { pollen } \\
\text { stain- } \\
\text { ability }\end{array}$} & \multirow{2}{*}{$\begin{array}{l}\text { Pods } \\
\text { produced } \\
\text { (range) }\end{array}$} \\
\hline & & Equal & Unequal & Laggards & Bridges & & \\
\hline \multicolumn{8}{|l|}{ Synthetic amphidiploid, $C_{1}$} \\
\hline \multicolumn{8}{|l|}{ Intercluster $(\mathrm{AB})$} \\
\hline batizocoi $\times$ correntina & 31 & 64.00 & 10.00 & 23.00 & 3.00 & 29 & 6 \\
\hline correntina $\times$ batizocoi & 5 & 80.00 & - & - & 20.00 & $29-78$ & 5 \\
\hline batizocoi $\times$ villosa & 16 & 63.00 & 19.00 & 18.00 & - & 75 & 5 \\
\hline \multicolumn{8}{|l|}{ Intracluster (AA) } \\
\hline sp. GKP $10038 \times$ duranensis & 20 & 50.00 & 35.00 & 15.00 & - & $41-69$ & $12-17$ \\
\hline sp. HLK $410 \times$ sp. GKP 10038 & 19 & 52.00 & 37.00 & 11.00 & - & 43 & $7-15$ \\
\hline sp. HLK $410 \times$ correntina & 14 & 65.00 & 21.00 & 14.00 & - & 57 & $13-19$ \\
\hline sp. HLK $410 \times$ villosa & 18 & 66.00 & 28.00 & 6.00 & - & 71 & $6-15$ \\
\hline $\begin{array}{l}\text { sp. HLK } 410 \times \\
\text { (chacoense } \times \text { cardenasii) }\end{array}$ & 12 & 42.00 & 50.00 & 8.00 & - & 79 & 3 \\
\hline correntina $\times$ sp. HLK 410 & 12 & 58.00 & 42.00 & - & - & 79 & 2 \\
\hline correntina $\times$ villosa & 28 & 36.00 & 25.00 & 39.00 & - & 71 & 0 \\
\hline villosa $\times$ sp. HLK 410 & 4 & 50.00 & 25.00 & 25.00 & - & 71 & 2 \\
\hline \multicolumn{8}{|l|}{ Synthetic amphidiploid, $C_{2}$} \\
\hline species GKP $10038 \times$ duranensis & 11 & 54.00 & - & 46.00 & - & - & $3-15$ \\
\hline villosa $\times$ batizocoi & 27 & 44.00 & 15 & 30.00 & 11 & 83 & $6-15$ \\
\hline
\end{tabular}

Table 3. Crossability between $A$. hypogaea and synthetic amphidiploids from diploid species of section Arachis genus $A$ rachis

\begin{tabular}{|c|c|c|c|c|c|c|}
\hline \multirow{2}{*}{$\begin{array}{l}\text { o hypogaea } \\
\text { Amphidiploids }\end{array}$} & \multicolumn{3}{|c|}{$\begin{array}{l}\text { A. hypogaea subspecies hypogaea } \\
\text { (Virginia) }\end{array}$} & \multicolumn{3}{|c|}{$\begin{array}{l}\text { A. hypogaea subspecies fastigiata } \\
\text { (Spanish) }\end{array}$} \\
\hline & $\begin{array}{l}\text { No. of } \\
\text { pollinations }\end{array}$ & $\begin{array}{l}\text { No. of } \\
\text { pods }\end{array}$ & $\begin{array}{l}\text { Pods } / 100 \\
\text { pollinations }\end{array}$ & $\begin{array}{l}\text { No. of } \\
\text { pollinations }\end{array}$ & $\begin{array}{l}\text { No. of } \\
\text { pods }\end{array}$ & $\begin{array}{l}\text { Pods } / 100 \\
\text { pollinations }\end{array}$ \\
\hline batizocoi $\times$ duranensis & 10 & 5 & 50 & - & - & - \\
\hline batizocoi $\times$ chacoense & 74 & 18 & 24 & 90 & 25 & 28 \\
\hline batizocoi $\times$ chacoense $e^{\text {a }}$ & - & - & - & 47 & 2 & 4 \\
\hline batizocoi $\times$ correntina & 11 & 2 & 18 & - & - & - \\
\hline correntina $\times$ batizocoi & - & - & - & 198 & 58 & 29 \\
\hline villosa $\times$ batizocoi & 82 & 18 & 22 & 208 & 60 & 29 \\
\hline sp. GKP $10038 \times$ sp. HLK 410 & - & - & - & 114 & 14 & 12 \\
\hline sp. GKP $10038 \times$ chacoense & - & - & - & 15 & 2 & 13 \\
\hline duranensis $\times$ sp. GKP 10038 & - & - & - & 118 & 37 & 31 \\
\hline duranensis $\times$ sp. HLK 410 & 10 & 5 & 50 & 31 & 4 & 13 \\
\hline duranensis $\times$ chacoense & 96 & 9 & 9 & 34 & 8 & 23 \\
\hline duranensis $\times$ cardenasii & - & - & - & 92 & 22 & 24 \\
\hline sp. HLK $410 \times$ sp. GKP 10038 & - & - & - & 222 & 21 & 9 \\
\hline sp. HLK $410 \times$ chacoense & - & - & - & 91 & 8 & 9 \\
\hline sp. HLK $410 \times$ correntina & - & - & - & 116 & 12 & 10 \\
\hline $\begin{array}{l}\text { sp. HLK } 410 \times \\
\quad(\text { chacoense } \times \text { cardenasii) }\end{array}$ & 164 & 32 & 20 & 17 & 5 & 29 \\
\hline correntina $\times$ sp. GKP 10038 & 56 & 13 & 23 & 15 & 0 & - \\
\hline correntina $\times$ sp. HLK 410 & 96 & 10 & 10 & 72 & 5 & 7 \\
\hline correntina $\times$ chacoense & 46 & 2 & 4 & 172 & 33 & 19 \\
\hline correntina $\times$ villosa & 229 & 54 & 23 & 236 & 50 & 21 \\
\hline $\begin{array}{l}\text { correntina } \times \\
\quad(\text { chacoense } \times \text { cardenasii })\end{array}$ & 163 & 27 & 17 & 102 & 19 & 19 \\
\hline villosa $\times$ duranensis & - & - & - & 131 & 25 & 19 \\
\hline villosa $\times$ sp. HLK 410 & 125 & 14 & 11 & 156 & 21 & 13 \\
\hline
\end{tabular}

a Reciprocal 

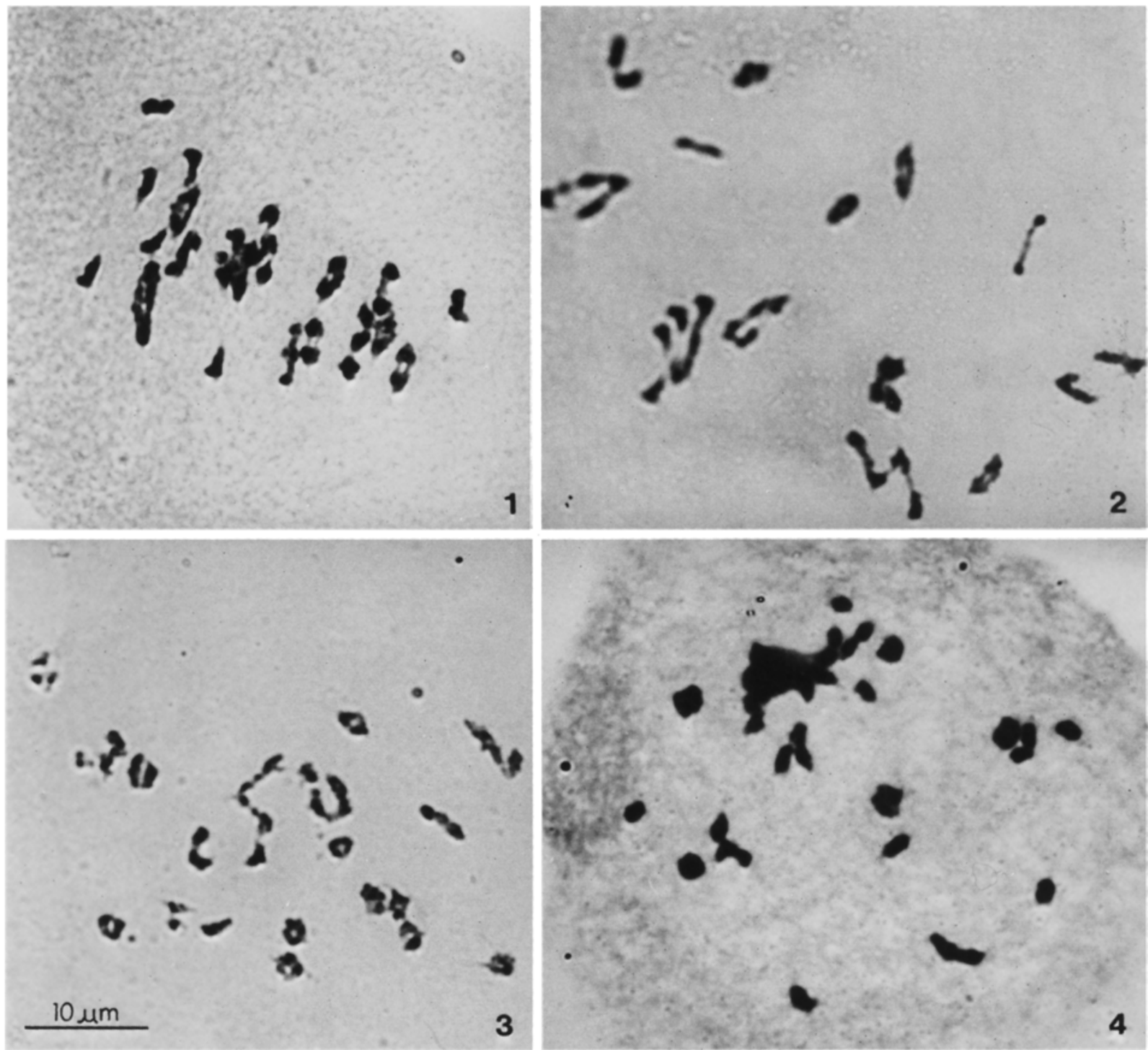

Figs. 1-4. Pollen mother cells at metaphase I in amphidiploids and in hybrids between $A$. hypogaea and amphidiploids: 1 $18 \mathrm{II}+1 \mathrm{IV}$ in an intercluster amphidiploid $A$. batizoco $\times A$. villosa. $212 \mathrm{II}+4 \mathrm{IV}$ in an intracluster amphidiploid $A$. duranensis $\times$ A. species GKP 10038.316 II +2 IV in A. hypogaea $\times(A$. batizocoi $\times A$. duranensis $) .416 \mathrm{II}+8 \mathrm{I}$ in $A$. hypogaea $\times(A$. duranensis $\times A$. chacoense)

\section{Synthetic amphidiploids, $C_{2}$}

Five intercluster and 11 intracluster amphidiploids were advanced in subsequent generations invariably involving an annual species as one of the parents. Plants in $C_{2}$ or subsequent generations were as vigorous as in $\mathrm{C}_{\mathbf{1}}$. Cytological analysis in these later generations revealed a significant decrease in univalents and often, though not always, an increase in bivalent association. A more normal orientation and synchronous movement of the chromosomes improved significantly their distribution at AI (Table 2). Consequently, the pollen and pod fertility of these plants also improved. Again, however, a greater variation in meiotic balance and pollen and pod fertility could be observed between progenies of different amphidiploids or within progenies of the same amphidiploid. This suggests a systematic selection to be rewarding. Gametes produced by more regular bivalent association have a better chance to produce a more stable progeny.

\section{A. hypogaea $\times$ synthetic amphidiploids, $F_{1}$}

The genomic relationships between $A$. hypogaea and the diploid wild species suggest that hybridization 
Table 4. Chromosome associations at metaphase $\mathrm{I}$ in $\mathrm{F}_{1}$ hybrids between $A$. hypogaea and synthetic amphidiploids of diploid species of section Arachis genus Arachis

\begin{tabular}{|c|c|c|c|c|c|c|c|}
\hline \multirow[t]{2}{*}{ Cross } & \multirow{2}{*}{$\begin{array}{l}\text { No. of } \\
\text { cells } \\
\text { analysed }\end{array}$} & \multicolumn{4}{|c|}{ Chromosome associations } & \multirow{2}{*}{$\begin{array}{l}\text { \% Pollen } \\
\text { stain- } \\
\text { ability } \\
\text { (range) }\end{array}$} & \multirow{2}{*}{$\begin{array}{l}\text { Pods } \\
\text { produced } \\
\text { (range) }\end{array}$} \\
\hline & & I & II & III & IV & & \\
\hline \multicolumn{8}{|l|}{ hypogaea $\times$ Synthetic amphidiploid, $F_{1}$} \\
\hline \multicolumn{8}{|l|}{ Intercluster $(\mathrm{AB})$} \\
\hline 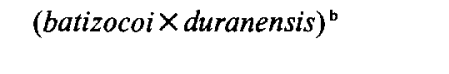 & 16 & $3.3 \pm 0.54$ & $16.4 \pm 0.57$ & $0.7 \pm 0.24$ & $0.4 \pm 0.13$ & $29-62$ & $\begin{array}{c}1-5 \\
(12-36)^{d}\end{array}$ \\
\hline 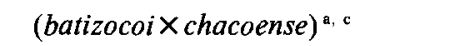 & 14 & $2.2 \pm 1.70$ & $16.2 \pm 1.82$ & $0.4 \pm 0.48$ & $0.9 \pm 0.80$ & - & 1 \\
\hline Reciprocal $^{\mathrm{c}}$ & 25 & $8.5 \pm 0.44$ & $13.4 \pm 0.36$ & $1.2 \pm 0.20$ & $0.3 \pm 0.11$ & 42 & 3 \\
\hline$(\text { batizocoi } \times \text { correntina })^{\mathbf{b}}$ & 16 & $4.9 \pm 0.47$ & $15.1 \pm 0.41$ & $0.5 \pm 0.16$ & $0.8 \pm 0.14$ & 37 & $2-4$ \\
\hline$(\text { correntina } \times \text { batizocoi })^{c}$ & 14 & $5.1 \pm 0.57$ & $14.4 \pm 1.03$ & $0.2 \pm 0.1$ & $1.4 \pm 0.4$ & $\begin{array}{c}40-57 \\
(52-70)^{d}\end{array}$ & $\begin{array}{c}2-4 \\
(1-57)^{d}\end{array}$ \\
\hline$(\text { villosa } \times \text { batizocoi })^{\mathbf{b}}$ & 25 & $4.7 \pm 0.43$ & $15.5 \pm 0.38$ & $0.8 \pm 0.16$ & $0.5 \pm 0.12$ & $\begin{array}{c}33-63 \\
(65-87)^{d}\end{array}$ & $\begin{array}{c}3-19 \\
(22-70)^{d}\end{array}$ \\
\hline \multicolumn{8}{|l|}{ Intracluster (AA) } \\
\hline$\left(_{\text {duranensis } \times \text { sp. GKP 10038) }}^{c}\right.$ & 20 & $9.5 \pm 0.42$ & $10.8 \pm 0.56$ & $1.1 \pm 0.23$ & $1.4 \pm 0.27$ & $43-64$ & $3-5$ \\
\hline 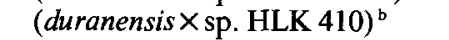 & 12 & $6.8 \pm 0.81$ & $14.5 \pm 0.34$ & $1.2 \pm 0.27$ & $0.2 \pm 0.11$ & 67 & \\
\hline$(\operatorname{sp~HLK~} 410 \times \text { sp. GKP } 10038)^{\mathfrak{c}}$ & 25 & $10.1 \pm 0.54$ & $11.5 \pm 0.60$ & $0.8 \pm 0.19$ & $0.9 \pm 0.19$ & $18-35$ & $34^{\mathrm{e}}$ \\
\hline${\text { (sp. HLK } 410 \times \text { chacoense) }^{c}}$ & 15 & $11.3 \pm 0.61$ & $11.0 \pm 0.54$ & $1.2 \pm 0.34$ & $0.9 \pm 0.22$ & 37 & 0 \\
\hline${\text { (correntina } \times \text { chacoense })^{\mathrm{b}}}^{\mathrm{b}}$ & 15 & $5.1 \pm 0.60$ & $15.0 \pm 0.44$ & $1.0 \pm 0.19$ & $0.5 \pm 0.13$ & - & 0 \\
\hline 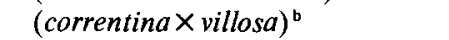 & 12 & $11.1 \pm 1.00$ & $11.3 \pm 0.66$ & $0.4 \pm 0.19$ & $1.3 \pm 0.33$ & 51 & 1 \\
\hline (villosa $\times$ duranensis) $^{\mathrm{c}}$ & 3 & $10.0 \pm 0.00$ & $13.0 \pm 1.15$ & $0.0 \pm 0.00$ & $1.0 \pm 0.58$ & $57-63$ & 2 \\
\hline$\left(\right.$ villosa $\times$ sp. HLK 410) ${ }^{b}$ & 14 & $7.9 \pm 1.02$ & $12.6 \pm 0.51$ & $1.6 \pm 0.31$ & $0.7 \pm 0.16$ & $17-55$ & $\begin{array}{l}0 \\
(0-14)^{d}\end{array}$ \\
\hline$\left(\right.$ villosa $\times \mathrm{sp}$. HLK 410) ${ }^{\mathrm{c}}$ & 35 & $8.7 \pm 0.41$ & $11.9 \pm 0.32$ & $1.1 \pm 0.15$ & $1.0 \pm 0.16$ & - & 0 \\
\hline $\begin{array}{l}\text { hypogaea } \times \text { Synthetic amphidiplo } \\
(\text { batizocoi } \times \text { duranensis })^{\mathrm{b}}\end{array}$ & $\begin{array}{r}F_{2} \\
13\end{array}$ & $2.9 \pm 0.60$ & $16.3 \pm 0.68$ & $0.7 \pm 0.26$ & $0.6 \pm 0.18$ & $40-62$ & 20 \\
\hline
\end{tabular}

a Cytology of $\mathrm{F}_{2}$ progeny

${ }^{b}$ Crossed with $A$. hypogaea subspecies hypogaea

' Crossed with $A$. hypogaea subspecies fastigiata

d ( ) pollen and pod fertility in $F_{4}$

'In 1 plant out of 3

between A. hypogaea and synthetic amphidiploids, besides providing a genetic bridge overcoming ploidy differences, would also provide (i) a combination of desirable features from at least two species, (ii) a more complete pairing between chromosomes of wild and cultivated species, and (iii) fertile hybrids from crosses between $A$. hypogaea and $\mathrm{AB}$ amphidiploids due to a complementary genomic constitution. The present cytogenetical analyses of hybrids between $A$. hypogaea and synthetic amphidiploids of diploid wild Arachis species, summarised in Tables 3 and 4, confirm these statements.

The intercluster amphidiploids more consistently produced a greater number of pods per 100 pollinations, probably due to their higher pollen fertility and better genomic complementation with $A$. hypogaea. The $F_{1}$ hybrids were vigorous and exhibited an intermediate morphology. The plant habit varied from runner to compact bunch type and most of them looked like $A$. hypogaea. The dominant feature of the parental wild diploid species, such as stem pigmentation, leaflet shape, margin, flower colour and resistance to rust, were expressed in the hybrids.

Cytological analysis of 15 of the 23 established $F_{1}$ hybrid combinations that included crosses between A. hypogaea and intra- as well as intercluster amphidiploids showed the theoretically expected pattern of chromosome pairing. Hybrids between A. hypogaea (AABB) and AABB amphidiploids had a significantly higher mean bivalent association than hybrids between A. hypogaea and AAAA amphidiploids (Table 4; Figs. 3 and 4). The more normal meiotic behaviour of the $A$. hypogaea $\times \mathrm{AABB}$ amphidiploid hybrids resulted in improved pollen and pod fertility as well as more offspring. This approach thus offers better breeding prospects for the transfer of genes from wild diploid species into $A$. hypogaea. Significantly lower bivalent associations in a reciprocal cross [ $A$. batizocoi $\times A$. chacoense) $\times A$. hypogaea] indicates the existence of cytoplasmic differences and that $A$. hypogaea should be 
used as the female parent for better pairing. Another aspect to consider is the dominant suspectibility to late leafspot of A. batizocoi, the lone representative of the B genome. This undesirable trait is transferred as a result of preferential autosyndetic (B-B) pairing. Selection of desirable recombinants with resistance to late leafspot and also to other important diseases, has not been possible despite the production of large hybrid populations through this route, involving various combinations of A and B genome species. Nevertheless, following this option lines resistant to rust have been bred from the crosses of $A$. batizocoi $\times A$.duranensis and A. correntina $\times$ A. batizocoi amphidiploids (Singh unpublished). It is likely that homoeologous pairing between A and B genomes may result in desirable recombinants from amphidiploids and selection at the amphidiploid level before crossing them to $A$. hypogaea may prove useful. Search for accessions of wild $A$. $b a$ tizocoi with resistance to late leafspot should be another approach.

Hybrids between $A$. hypogaea and AAAA amphidiploids are able to form more than 10 bivalents and a few multivalents (Table 4). Such a configuration indicates not only a high intragenomic pairing within the A group but that intergenomic homoeologous pairing (A-B) also occurs. Gene transfers from AAAA amphidiploids may thus involve not only the A but also the $\mathrm{B}$ genome of $A$. hypogaea. At the ICRISAT Center, several hybrids, such as $A$. hypogaea $\times(A$. correntina $\times A$. chacoense) and $A$. hypogaea $\times(A$. species GKP $10038 \times$ $A$. species HLK 410), involving wild species resistant to rust and/or leafspot diseases, have led to the production and selection of segregants resistant to both rust and late leafspot. Further advancement through selection towards genetic uniformity and more acceptable agronomic performance is in progress.

Among the $A$. hypogaea $\times$ AAAA amphidiploids, the combinations $A$. hypogaea $\times(A$. correntina $\times A$. chacoense) and $A$. hypogaea $\times(A$. duranensis $\times A$. species HLK 410) proved to have a significantly high bivalent association, indicating a probable genetic control of pairing in certain intracluster combinations (Table 4). However, the number of cells scored in these hybrids is small and further investigations are required before any definite conclusion can be made. The identical chromosome association in crosses between $A$. villosa $\times A$. species HLK 410 amphidiploids and cultivars representing both subspecies of $A$. hypogaea suggests an identical genomic constitution of the latter.

\section{A. hypogaea $\times$ synthetic amphidiploid, $F_{2}$}

The meiotic configuration proved to be nearly identical at $F_{1}$ and $F_{2}$ of the one combination that was analyzed (Table 4). A successive improvement in pollen and pod fertility could, however, be recorded in hybrid progenies advanced into subsequent generations, but with considerable variation between families. This variation may be attributed to different levels of auto- and allosyndetic pairing involving $A$. hypogaea chromosomes and loss of wild species chromosomes during meiosis. A few $\mathrm{F}_{4}$ plants of $A$. hypogaea $\times(A$. batizocoi $\times A$. duranensis) were able to produce two-seeded pods as in $A$. hypogaea.

The present investigation of amphidiploids and their hybrids with $A$. hypogaea have helped in highlighting the advantages and disadvantages of using amphiploidy as a way to transfer certain traits from the wild Arachis species into the cultivated groundnut. In addition, a better understanding of the genomic and species interrelations in Arachis is gained.

Acknowledgements. The author is indebted to Mr. R.W. Gibbons, Leader, Groundnut Improvement Program, ICRISAT for his critical reading of the manuscript and suggestions. Thanks are also due to Mrs. Kamal Wankhede and A. Venkateshwar for technical assistance and to Mrs. Sashikala for secretarial assistance.

\section{References}

Abdou YAM, Gregory WC, Cooper WE (1974) Sources and nature of resistance to Cercospora arachidicola Hori and Cercosporidium personatum (Berk and Curt.) Deighton in Arachis species. Peanut Sci 1:6-11

Amin PW (1985) Resistance of wild species of groundnut to insect and mite pests. In: ICRISAT (International Crops Research Institute for the Semi-Arid Tropics). Proc Int Workshop Cytogenet Arachis. ICRISAT Center, Patancheru AP, India, pp 57-60

Cochran WC, Cox GM (1957) Experimental designs. Wiley and Sons, New York, pp 95-102

Gardner MED, Stalker HT (1983) Cytology and leafspot resistance of section Arachis amphiploids and their hybrids with Arachis hypogaea. Crop Sci 23: 1069-1074

Husted L (1936) Cytological studies of the peanut, Arachis L. 2. Chromosome number, morphology and behaviour and their application to the origin of cultivated forms. Cytologia 7:396-423

Moss JP (1980) Wild species in the improvement of groundnuts. In: Summerfield RJ, Bunting AH (eds) Advances in legume science. Royal Botanic Garden, Kew (England), pp 525-535

Singh AK (1985) Genetic introgression from compatible Arachis species into groundnut. In: ICRISAT (International Crops Research Institute for the Semi-Arid Tropics). Proc Int Workshop Cytogenet Arachis. ICRISAT Center, Patancheru AP, India, pp 107-117

Singh AK (1986) Utilization of wild relatives in genetic improvement of Arachis hypogaea L. 7. Autotetraploid production and prospects in interspecific breeding. Theor Appl Genet 72: 164-169

Singh AK, Moss JP (1982) Utilization of wild relatives in genetic improvement of Arachis hypogaea L. 2. Chromosome complements of species of section Arachis. Theor Appl Genet 61:305-314 
Singh AK, Moss JP (1984) Utilization of wild relatives in genetic improvement of Arachis hypogaea L. 5. Genome analysis in section Arachis and its implications in gene transfer. Theor Appl Genet 68:355-364

Smartt J, Gregory WC, Gregory MP (1978) The genomes of Arachis hypogaea. 1. The implications in interspecific breeding. Euphytica 27:677-680

Stalker HT, Wynne JC (1979) Cytology of interspecific hybrids in section Arachis of peanuts. Peanut Sci 6:110-114
Subrahmanyam P, Ghanekar AM, Knolt BL, Reddy DVR, McDonald D (1985) Resistance to groundnut diseases in wild Arachis species. In: ICRISAT (International Crops Research Institute for the Semi-Arid Tropics) Proc Int Workshop Cytogenet Arachis. ICRISAT Center, Patancheru, AP, India, pp 49-55 